\title{
Health hazards of electronic cigarettes and their utility in smoking cessation
}

\author{
Matthew A. Steliga, MD, FACS
}

Feature Editor's Introduction-There are more than 1 billion smokers in the world, and the clinical practices of thoracic surgeons are some of those most highly enriched for smokers. Reciprocally, for many smokers, a thoracic surgeon is the first provider seen at a time when they are genuinely interested in quitting smoking. Further, many of these patients require pulmonary resection, and the rate of surgical morbidity is more than double for patients who do not abstain from smoking at least 4 weeks before surgery. The encounter with a thoracic surgeon is therefore a golden "teaching moment" for which appropriate intervention can have meaningful short-term and long-term impact, and it is important for thoracic surgeons be informed about the science of addiction and how to implement effective therapies for smoking cessation. Because of their relatively decreased carcinogens and toxins compared with combustible cigarettes, electronic cigarettes have been suggested as a platform for cessation of traditional cigarettes; however, they bring with them new and incompletely understood risks. Questions about the safety of electronic cigarettes and their efficacy in smoking cessation are common in thoracic surgery clinics, and recent randomized trials have investigated their utility for this purpose. The material included in the following Feature Expert Opinion article will inform the thoracic surgeon of these recent data and expand the scope of their clinical practice.

\section{Bryan M. Burt, MD}

Smoking is the greatest controllable risk factor for developing many diseases treated by cardiac, vascular, and thoracic surgeons. Unsurprisingly, patient populations in cardiac, vascular, and thoracic clinical settings typically have higher smoking rates than those of the general public. This is a common challenge we face on a daily basis. Patients, families, physicians, and others may feel a sense of futility regarding cessation, because the patient already has developed a smoking-related disease; however, cessation at any point does have significant benefits. ${ }^{1}$ Studies have demonstrated improved cancer survival associated with cessation, ${ }^{2}$ and significant increased costs of cancer

\footnotetext{
From the Division of Thoracic Surgery, University of Arkansas for Medical Sciences, Little Rock, Ark.

Received for publication May 2, 2019; revisions received Oct 30, 2020; accepted for publication Nov 11, 2020; available ahead of print Jan 13, 2021.

Address for reprints: Matthew A. Steliga, MD, FACS, Division of Thoracic Surgery, University of Arkansas for Medical Sciences, 4301 W Markham Ave \#725, Little Rock, AR (E-mail: MASteliga@uams.edu).

J Thorac Cardiovasc Surg 2022;163:307-10

$0022-5223 / \$ 36.00$

Copyright () 2021 by The American Association for Thoracic Surgery

https://doi.org/10.1016/j.jtcvs.2020.11.180
}

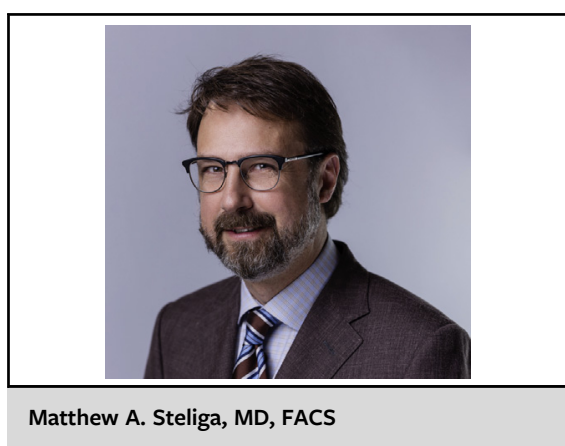

\begin{abstract}
CENTRAL MESSAGE
This Invited Expert Opinion discusses evidence regarding the impact of e-cigarettes on smoking cessation, potential harms of e-cigarettes, and areas of uncertainty requiring further investigation.
\end{abstract}

See Commentaries on pages 311 and 312.

treatment failure due to continued smoking have been demonstrated. ${ }^{3}$ Cessation can decrease perioperative complications, ${ }^{4}$ and most who are currently smoking want to quit. ${ }^{5}$ Nicotine is incredibly addictive, and this addiction contributes to poor cessation rates, with frequent relapses after quitting. Multiple different strategies exist to help patients quit smoking, and there is no one method that works best for all patients. ${ }^{5}$ Because of difficulty with cessation, perceived lack of benefit, and unfamiliarity with cessation resources, many patients may not receive adequate support to quit. For example, self-reported survey data of lung cancer physicians from multiple disciplines showed that even in settings with experts who think cessation is important, many do not offer assistance to patients regarding smoking cessation. ${ }^{6}$ Smoking cessation resources can be integrated into a clinical workflow in surgical settings to deliver evidence-based support to patients. ${ }^{7}$ Approved pharmacotherapy that has been demonstrated to assist with cessation includes varenicline, bupropion, and pharmacologic nicotine replacement therapy (NRT). NRT in this context refers to the pharmacologic NRT and does not indicate electronic cigarette (e-cigarette) use even though e-cigarettes do contain nicotine. The only long-acting form of NRT is the transdermal nicotine patch, whereas short-acting 
forms include gum, lozenge, nasal spray, and a nonelectronic pharmacologic inhaler. A Cochrane review found varenicline was the most effective single drug for cessation and equivalent to combination (patch plus short-acting) NRT. ${ }^{8}$ However, even when patients do receive evidencebased pharmacotherapy combined with behavioral counseling, more than half may fail approved methods.

\section{ELECTRONIC CIGARETTES}

E-cigarettes were initially developed as a cessation aid, but there are multiple products with different nicotine concentrations and various other chemical constituents. Different styles of devices resemble conventional cigarettes, cigars, or even hookahs. Some newer-generation devices have modifiable electronic controls. In regulatory documentation and scientific literature, they may be referred to as "electronic nicotine delivery systems," but because "e-cigarette" is the most common terminology used in our clinical setting, this discussion will use the term "e-cigarettes." From a toxicology standpoint, e-cigarettes have been demonstrated to contain decreased carcinogens and toxins compared with combustible cigarettes. ${ }^{9}$ The nicotine content from an e-cigarette may be variable, ranging from more than combustible cigarettes to less, to none..$^{10}$ As a heterogeneous group of devices, e-cigarettes are not standardized and have not been subjected to rigors of scientific testing for approval as a standardized drug with a predictable dose to treat a medical condition. From a regulatory standpoint, e-cigarettes are not a pharmacologic agent cleared by the US Food and Drug Administration to treat nicotine addiction. Despite the lack of approval as a drug to treat a condition, e-cigarettes do deliver nicotine to the consumer and have been used by many as part of a cessation plan. A longitudinal online survey of US smokers demonstrated that the most common method attempted for cessation $(65.3 \%)$ was abrupt cessation ("cold turkey"), and the second most common method $(62.0 \%)$ was tapering or reducing the number of cigarettes smoked. ${ }^{11}$ Use of e-cigarettes at the same time as smoking combustible cigarettes (also known as "dual use") was reported by $35.3 \%$ of responders, and complete substitution of an e-cigarette for the combustible cigarettes was reported by $24.7 \%$. Only $25.4 \%$ reported using NRT, and $12.2 \%$ used bupropion or varenicline. ${ }^{11}$ Many reported attempting multiple methods, but use of an e-cigarette was more common than approved pharmacotherapy. Data from the United Kingdom also demonstrate an increase in the popularity of e-cigarettes that was correlated with increased success with quit attempts. ${ }^{12}$ Popularity of a cessation method certainly does not indicate efficacy, but many trials have been done to compare e-cigarettes with conventional cessation methods. A Cochrane review of e-cigarettes used for cessation concluded that nicotine-containing e-cigarettes aided cessation above placebo, but could not determine superiority over conventional approved methods including NRT. ${ }^{13}$ A systematic review concluded that the studies to date were inconclusive because of low quality, but overall the literature suggested e-cigarettes may be helpful for some smokers for quitting or reducing smoking. ${ }^{14}$ Recently, a randomized controlled trial by Hajek and colleagues ${ }^{15}$ demonstrated e-cigarettes to be superior to NRT in a structured program coupled with behavioral counseling support. In their trial, $18.0 \%$ in the e-cigarette group remained abstinent from combustible cigarettes at 1 year compared with $9.9 \%$ in the NRT group. In this trial, the NRT group used whichever product they preferred, and combination NRT (patch with short-acting gum or lozenge) was encouraged. Both groups received structured behavioral counseling. Interestingly, of those who quit with NRT, only $9 \%(4 / 44)$ were still using the NRT 1 year later, and the rest were nicotine free. Of those who quit with the e-cigarettes, $80 \%(63 / 79)$ were still using the e-cigarette at the 1-year point, suggesting that the e-cigarettes had significant potential for addiction. ${ }^{15}$ Generalization from this structured controlled environment to all use of e-cigarette devices may not be valid, but these data demonstrate that e-cigarettes can benefit patients in some circumstances, particularly in the context of a program with structured behavioral counseling.

\section{POTENTIAL HARMS}

Because e-cigarettes are a relatively new technology, we simply do not have decades of data on users to determine long-term health impacts, but acute injuries and intoxications can occur and could be fairly severe. Burns or explosions of e-cigarettes often require surgery and skin grafting, and even death has been reported due to a malfunctioning device. ${ }^{16}$ Death due to ingestion of the nicotine-containing liquid has also been reported in a child. ${ }^{17}$ Because nicotine can be absorbed transdermally, children may experience intoxication from contact with improperly stored liquid. Severe harms are possible but incredibly rare. What remains a greater question is potential long-term harm. Tumorigenic potential of ecigarette exposure was demonstrated in a murine model in which e-cigarette exposure was linked to lung and bladder cancer. ${ }^{18}$ A murine model has also demonstrated emphysema changes from e-cigarette exposure as well. ${ }^{19}$ It is unknown whether this would be clinically seen in humans with the current products. There have also been multiple reports of ecigarette and vaping-related lung injury in 2019 and $2020 .{ }^{20}$ However, many, but not all, of these cases were linked to e-cigarettes containing tetrahydrocannabinol or other substances besides nicotine. ${ }^{20}$ Although e-cigarettes contain less toxins than conventional cigarettes, they do contain substances besides simply "nicotine and water vapor." Glycerol, propylene glycol, and flavorings added to the liquid are cleared as safe for ingestion, but not for heating and inhalation. Clearly, there are many foods and drugs that are safe to ingest but by no means does that indicate they are harmless if heated and then inhaled. Some flavorings such as diacetyl have been implicated in the development of bronchiolitis obliterans (also known as "popcorn lung") 
demonstrated radiographically and on open lung biopsy after e-cigarette use. ${ }^{21}$ These types of changes may be chronic and slower to develop over years because they appear to be a different pattern of injury than the acute changes seen with the e-cigarette and vaping-related lung injury cases, which have a more acute presentation. The recent global pandemic of Coronavirus Disease 2019 has led to increased awareness of respiratory health, and although no long-term data exist about vaping and Coronavirus Disease 2019 risk, it appears to be a motivator that increases some to seek help quitting through quit lines and by increased quit attempts. ${ }^{22}$ At a time when there is increased concern about transmission of viral respiratory illness, it is unknown if e-cigarettes could pose increased risk through increased hand-mouth contact, sharing devices, or respiratory droplets and aerosols.

E-cigarettes are marketed as a cessation aid, and although they may help some people quit nicotine altogether, the reality is that many do not use counseling support or a structured quit plan and may become dual-users (e-cigarette plus combustible cigarette) or long-term e-cigarette users. Dual-use might help if there is a structured tapering, but unfortunately, there is concern that without a plan, dual-users may often use e-cigarettes in areas where they cannot smoke, still smoke when they can, and increase overall daily nicotine intake that could hamper cessation. ${ }^{23}$ The combination of the addictive potential of e-cigarettes and the perception of safety may lead many to become long-time users, as illustrated by the $80 \%$ of those who quit with e-cigarettes, still using the devices 1 year later. ${ }^{15}$ If e-cigarettes are used as cessation aids, there should be a structured goal to quit combustible cigarettes as quickly as possible and then make a structured plan with a timeline to quit e-cigarettes, because the long-term use of them may contain risks that are not fully understood. Overall, there are both rare acute risks and potential unknown long-term risks with chronic use, but there are also clearly known risks with smoking cigarettes. If e-cigarettes can improve quit rates, there may be potential benefit as long as there is not ongoing use and associated chronic harm.

\section{KEY POINTS REGARDING E-CIGARETTES FOR CESSATION}

- Potential exists for long-term addiction to e-cigarettes. ${ }^{15}$

- Explosions, burns, and poisonings can be serious or even fatal but are exceedingly rare. ${ }^{16,17}$

- Development of cancer or chronic lung disease has not been demonstrated in humans, but some animal model data suggest possible mechanisms. ${ }^{18,19}$

- Acute lung injuries have been most often, but not always, linked to e-cigarettes containing tetrahydrocannabinol and other substances. ${ }^{20}$

- Long-term use of pharmacologic nicotine appears safe, but effects of long-term use of e-cigarettes are not known because of the novelty of the technology.
- Chronic lung disease such as bronchiolitis obliterans is not common but is possible and not reversible. ${ }^{21}$

- E-cigarettes do contain less carcinogens and other toxins compared with tobacco cigarettes, and some health systems such as the UK's National Health Service support it as an option for cessation. ${ }^{24}$

- Some patients may be able to quit with an e-cigarette, and if so, they should have a structured plan and counseling by an expert in cessation.

\section{SOCIETAL ISSUES}

The focus of this Expert Opinion is to discuss e-cigarette use in the context of patients who want to quit smoking. It is not possible to dissect out all the issues related to the e-cigarette industry, which has significant ownership by the tobacco industry, and the potential for marketing to youth and nicotine-naïve young adults. It is concerning that many youth whose first exposure to nicotine is in the form of e-cigarettes that could lead to increased rates of smoking combustible cigarettes. ${ }^{25}$ In select cases, e-cigarettes may help some who struggle to quit, but at the same time, it may feel conflicting to advocate for and support an industry entangled with the tobacco industry, which spends a great deal of resources on advertising and lobbying to undermine current tobacco control efforts. It should be reasonable to explore ways that e-cigarettes can genuinely help patients, but still have strong enforceable policy to prevent youth use and stronger tobacco-control policies to eliminate combustible cigarettes, while preventing novel products from increasing in popularity. Unfortunately, the field is an evolving landscape of novel products. Heated tobacco products (HTPs) are currently available in Japan and parts of Canada, and recently have been marketed in the United States. Similar to e-cigarettes, HTPs are electronic devices that produce heat, but rather than heating a liquid, HTPs warm but do not actually combust sticks of compressed tobacco infused with flavorings and additives. The tobacco industry touts HTP as a reduced risk product, but clearly it is an addictive product with some risk that we need to be aware of as looming on the horizon.

\section{ADDRESSING CLINICAL SCENARIOS}

As clinicians, we are often faced with certain questions from our patients, their families, and others. It is difficult to make clear recommendations with incomplete and often conflicting data. There are a few principles that would likely be agreed upon by most clinicians and researchers. First, smoking is addictive and harmful, particularly for our surgical patients. There are benefits to cessation at any point. Evidencebased strategies include pharmacotherapy combined with counseling. E-cigarettes may help some people quit smoking. E-cigarettes contain less toxins than cigarettes, but they may be addictive and are not completely harmless. With these concepts as a foundation, our tobacco-cessation program 
has specific standardized approaches for different categories of patients.

- All patients actively smoking must be advised by the physician of the benefits of quitting and referred to a cessation expert, either through a quit line or in person for counseling and pharmacotherapy.

- Those who have failed multiple approved methods and strongly want to attempt cessation with an e-cigarette should quit tobacco cigarettes altogether and make a plan for tapering off the e-cigarette. E-cigarette use for cessation must be combined with behavioral counseling.

- Patients who are currently dual-users must make a structured plan to eliminate combustible cigarettes as quickly as possible and then a plan to address e-cigarette use.

- For the patient solely using the e-cigarette and unable to quit, we explore pharmacotherapy, typically varenicline or combination NRT, to help them eliminate the e-cigarette in conjunction with counseling.

\section{CONCLUSIONS}

Overall, e-cigarettes are a constantly growing, evolving field. Higher nicotine concentrations may aid cessation, but they have the potential for stronger addiction. There is much to be learned about how best to address e-cigarettes in the context of cessation. Certainly, there is benefit for some and reduced risk compared with cigarettes, but e-cigarettes are not completely without risk. The use of e-cigarettes should not be viewed as first-line therapy when there is clear, strong evidence for efficacy and safety of pharmacotherapy and counseling. If evidence-based support fails, they could be used as one potential stepping stone toward freedom from nicotine, but not an ultimate destination, because the long-term risks are still not known.

\section{Conflict of Interest Statement}

The author reported no conflicts of interest.

The Journal policy requires editors and reviewers to disclose conflicts of interest and to decline handling or reviewing manuscripts for which they may have a conflict of interest. The editors and reviewers of this article have no conflicts of interest.

\section{References}

1. Substance Abuse and Mental Health Services Administration (US), Office of the Surgeon General (US). Smoking Cessation: A Report of the Surgeon General, The Health Benefits of Smoking Cessation (Ch 4). Washington, DC: US Department of Health and Human Services; 2020.

2. Amato KA, Hyland A, Reed R, Mahoney MC, Marshall J, Giovino G, et al. Tobacco cessation may improve lung cancer patient survival. J Thorac Oncol. 2015; 10:1014-9

3. Warren GW, Cartmell KB, Garrett-Mayer E, Salloum RG, Cummings KM Attributable failure of first-line cancer treatment and incremental costs associated with smoking by patients with cancer. JAMA Netw Open. 2019;2:e191703.
4. Wong J, Lam DP, Abrishami A, Chan MT, Chung F. Short-term preoperative smoking cessation and postoperative complications: a systematic review and meta-analysis. Can J Anaesth. 2012;59:268-79.

5. Tobacco Use and Dependence Guideline Panel. Treating Tobacco Use and Dependence: 2008 Update. Rockville, MD: US Department of Health and Human Services; 2008. Available at: https://www.ncbi.nlm.nih.gov/books/ NBK63952/. Accessed February 11, 2021.

6. Warren GW, Marshall JR, Cummings KM, Toll B, Gritz ER, Hutson A, et al; IASLC Tobacco Control and Smoking Cessation Committee. Practice patterns and perceptions of thoracic oncology providers on tobacco use and cessation in cancer patients. J Thorac Oncol. 2013;8:543-8.

7. Steliga MA. Smoking cessation in clinical practice: how to get patients to stop. Semin Thorac Cardiovasc Surg. 2018;30:87-91

8. Cahill K, Stevens S, Perera R, Lancaster T. Pharmacological interventions for smoking cessation: an overview and network meta-analysis. Cochrane Database Syst Rev. 2013;5:CD009329.

9. Goniewicz ML, Knysak J, Gawron M, Kosmider L, Sobczak A, Kurek J, et al. Levels of selected carcinogens and toxicants in vapour from electronic cigarettes. Tob Control. 2014;23:133-9.

10. Ramôa CP, Hiler MM, Spindle TR, Lopez AA, Karaoghlanian N, Lipato T, et al. Electronic cigarette nicotine delivery can exceed that of combustible cigarettes: a preliminary report. Tob Control. 2016;25:e6-9.

11. Caraballo RS, Shafer PR, Patel D, Davis KC, McAfee TA. Peer reviewed: quit methods used by US adult cigarette smokers, 2014-2016. Prev Chronic Dis. 2017; 14:E32.

12. Beard E, West R, Michie S, Brown J. Association between electronic cigarette use and changes in quit attempts, success of quit attempts, use of smoking cessation pharmacotherapy, and use of stop smoking services in England: time series analysis of population trends. BMJ. 2016;354:i4645.

13. Hartmann-Boyce J, McRobbie H, Bullen C, Begh R, Stead LF, Hajek P. Electronic cigarettes for smoking cessation. Cochrane Database Syst Rev. 2016;9:CD010216.

14. Malas M, van der Tempel J, Schwartz R, Minichiello A, Lightfoot C, Noormohamed A, et al. Electronic cigarettes for smoking cessation: a systematic review. Nicotine Tob Res. 2016;18:1926-36.

15. Hajek P, Phillips-Waller A, Przulj D, Pesola F, Myers Smith K, Bisal N, et al. A randomized trial of e-cigarettes versus nicotine-replacement therapy. $N$ Engl J Med. 2019;380:629-37.

16. Jiwani AZ, Williams JF, Rizzo JA, Chung KK, King BT, Cancio LC. Thermal injury patterns associated with electronic cigarettes. Int J Burns Trauma. 2017;7:1.

17. Seo AD, Kim DC, Yu HJ, Kang MJ. Accidental ingestion of E-cigarette liquid nicotine in a 15-month-old child: an infant mortality case of nicotine intoxication. Korean J Pediatr. 2016;59:490.

18. Lee HW, Park SH, Weng MW, Wang HT, Huang WC, Lepor H, et al. E-cigarette smoke damages DNA and reduces repair activity in mouse lung, heart, and bladder as well as in human lung and bladder cells. Proc Natl Acad Sci U S A. 2018;115:E1560-9.

19. Garcia-Arcos I, Geraghty P, Baumlin N, Campos M, Dabo AJ, Jundi B, et al. Chronic electronic cigarette exposure in mice induces features of COPD in a nicotine-dependent manner. Thorax. 2016;71:1119-29.

20. Navon L, Jones CM, Ghinai I, King BA, Briss PA, Hacker KA, et al. Risk factors for e-cigarette, or vaping, product use-associated lung injury (EVALI) among adults who use e-cigarette, or vaping, products-Illinois, July-October 2019. MMWR Morb Mortal Wkly Rep. 2019;68:1034.

21. Flower M, Nandakumar L, Singh M, Wyld D, Windsor M, Fielding D. Respiratory bronchiolitis-associated interstitial lung disease secondary to electronic nicotine delivery system use confirmed with open lung biopsy. Respirol Case Rep. 2017;5:e00230.

22. Klemperer EM, West JC, Peasley-Miklus C, Villanti AC. Change in tobacco and electronic cigarette use and motivation to quit in response to COVID-19. Nicotine Tob Res. 2020;22:1662-3.

23. Orellana-Barrios MA, Payne D, Mulkey Z, Nugent K. Electronic cigarettes-a narrative review for clinicians. Am J Med. 2015;128:674-81.

24. National Health System UK. Using e-cigarettes to stop smoking. Available at: www.nhs.uk/live-well/quit-smoking/using-e-cigarettes-to-stop-smoking/. Accessed March 29, 2019

25. Bold KW, Kong G, Camenga DR, Simon P, Cavallo DA, Morean ME, et al. Trajectories of e-cigarette and conventional cigarette use among youth. Pediatrics. 2018;141:e20171832.

Key Words: electronic cigarette, smoking cessation, tobacco 